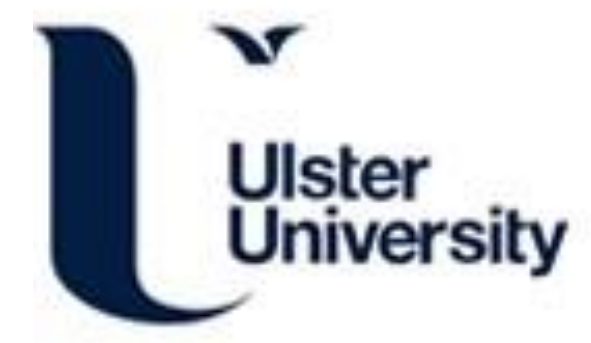

\title{
View factor in cone calorimeter testing
}

Kang, S., Choi, S-K., \& Choi, J. Y. (2016). View factor in cone calorimeter testing. International Journal of Heat and Mass Transfer, 93, 217-227. https://doi.org/10.1016/j.ijheatmasstransfer.2015.09.067

Link to publication record in Ulster University Research Portal

\section{Published in:}

International Journal of Heat and Mass Transfer

Publication Status:

Published (in print/issue): 01/02/2016

DOI:

10.1016/j.ijheatmasstransfer.2015.09.067

\section{Document Version}

Author Accepted version

\section{General rights}

Copyright for the publications made accessible via Ulster University's Research Portal is retained by the author(s) and / or other copyright owners and it is a condition of accessing these publications that users recognise and abide by the legal requirements associated with these rights.

\section{Take down policy}

The Research Portal is Ulster University's institutional repository that provides access to Ulster's research outputs. Every effort has been made to ensure that content in the Research Portal does not infringe any person's rights, or applicable UK laws. If you discover content in the Research Portal that you believe breaches copyright or violates any law, please contact pure-support@ulster.ac.uk. 


\title{
View factor in cone calorimeter testing
}

\author{
Sungwook Kang ${ }^{a}$ Sengkwan Choi ${ }^{a, *}$ and J.Yoon Choi ${ }^{b}$ \\ ${ }^{\text {a }}$ School of the Built Environment, Ulster University, Newtownabbey, Co.Antrim, BT37 0QB, \\ UK \\ ${ }^{\mathrm{b}}$ Fire Safety Team, Division of Built Environment, Korea Conformity Laboratories, Ochang, \\ Chungchongbukdo, Republic of Korea
}

\begin{abstract}
This work focuses on algebraic derivations of geometric view factors (i) from plane element to interior of truncated cone in parallel configuration; (ii) from plane element to segment of interior of truncated cone in perpendicular configuration, to clarify irradiance-related uncertainties generated in cone calorimeter tests on intumescent-type fire resistant systems. Since such specimens undergo moving boundaries and perimeter surface exposures in the course of the bench-scaled fire tests, it is inevitable to encounter (i) irradiance intensifications on their top boundaries and (ii) irradiance influxes on their perimeter areas, which have not been reflected in conventional approaches. These irradiance-related issues can be solved by calculating diffuse view factors. Their derivations are achieved by using the contour integration method and verified by existing literature and direct measurements. The calculations are presented by graphical representations obtained through a process of mapping. This theoretical approach enables one to clarify the exact quantity of irradiance at any position under the heater, and thus to quantitatively analyse the resultant impacts of (i) non-uniform irradiance dispersions and (ii) non-consistent thermal loads occurring during the tests, on the quantification of radiation absorption. The findings demonstrate that discrepancies between exact calculations and conventional approximations, induced by these effects, are appreciable and hence should not be neglected in such quantifications. The derived formulae can be applied in solving radiation issues arising with analogous geometries, and the particulars in terms of irradiance can also promote the subsequent assessment of thermal behaviours of any specimen experiencing geometrical changes during cone calorimeter tests.
\end{abstract}

Keywords: Thermal radiation; Irradiance; View factor; Cone calorimeter; Contour integration method

\footnotetext{
* Corresponding author.

E-mail address: s.choi@ulster.ac.uk (Sengkwan Choi)
} 


\begin{tabular}{|c|c|c|c|}
\hline \multicolumn{4}{|c|}{ Nomenclature } \\
\hline$A$ & surface area & \multicolumn{2}{|c|}{ Greek symbols } \\
\hline$b, q, s, t$ & spacing parameters & $\alpha$ & absorptivity \\
\hline$C$ & contour & $\beta, \theta, \varphi, \phi$ & $\begin{array}{l}\text { angular integration } \\
\text { variables }\end{array}$ \\
\hline$F$ & view factor & $\rho$ & spacing parameter \\
\hline$h, h_{2}$ & $\begin{array}{l}\text { vertical spacing parameter from } \\
\text { cone baseplate to top surface of } \\
\text { specimen }\end{array}$ & $\sigma$ & $\begin{array}{l}\text { Stefan-Boltzmann constant } \\
\left(\mathrm{W} / \mathrm{m}^{2} \mathrm{~K}^{4}\right)\end{array}$ \\
\hline$h_{4}$ & $\begin{array}{l}\text { vertical spacing parameter from } \\
\text { cone upperplate to top surface of } \\
\text { specimen }\end{array}$ & $\omega$ & solid angle \\
\hline$H$ & height of conical heater & & \\
\hline$k$ & coverage factor & Subscripts & \\
\hline$\ell, m, n$ & direction cosines & $a$ & arc \\
\hline$\vec{n}$ & normal vector & $a b s$ & absorbed \\
\hline$p$ & $\begin{array}{l}\text { horizontal spacing parameter from } \\
\text { the origin of the coordinate system }\end{array}$ & emit & emitted \\
\hline$P$ & general point upon contours & $g$ & heat flux gauge \\
\hline$\dot{q}^{\prime \prime}$ & radiant heat flux $\left(\mathrm{W} / \mathrm{m}^{2}\right)$ & heater & conical heater \\
\hline$r$ & radial dimension & $\ell$ & line \\
\hline$u$ & standard uncertainty & $s$ & specimen \\
\hline$u_{c}$ & combined uncertainty & & \\
\hline$U$ & expanded uncertainty & & \\
\hline$x, y, z$ & Cartesian coordinates & & \\
\hline
\end{tabular}




\section{Introduction}

A bench-scaled cone calorimeter, standardised in BS 476-15 [1], has been widely used to test flammability of various condensed materials in the field of fire safety engineering. This apparatus facilitates regulating a radiant heat flux and is capable of imposing a consistent thermal load on given specimens during tests, which are important aspects when creating a stable fire test environment. The steady heating condition has extended its application, beyond the conventional purpose, to evaluating thermal behaviours and performances of intumescent-type fire retardant systems since the early 2000s [2-10]. With this extended application, doubts have recently arisen about whether thermal boundaries of the polymeric samples tested with this instrument are either adequately clarified or understood. This is because this type of material exhibits anisotropic volume progressions, induced by thermochemical decompositions, by up to several tens of times its dry film thickness when subjected to a heat. In cone calorimeter tests on such materials, the thermal reaction leads to, primarily having their top boundaries moved toward the spatially stationary conical heater, and secondarily having their perimeter surfaces progressively extended in the z-direction and newly exposed to the heating element, as described in Fig. 1.

The quantity of incident radiant flux on given specimens is highly critical in solving heat-related issues of flammability, thermal behaviours and performances [11]. In this bench-scaled fire test, irradiance is typically estimated by physical measurements using a heat flux probe (e.g., Schmidt-Boelter gauge) placed along the cone axis through the central point of test samples in a calibration stage [12]. Up until now, a single value measured in this initial stage has been interpreted as a constant thermal load in the entire course of tests, even on intumescent-type materials. However, this conventional approach does not reflect

- the intensification of irradiance on the top surface of specimens, developed as the boundaries progressively approach the heat source. The alteration of thermal loads in the process of testing can lead to an underestimate of the amount of heat absorption, by up to approximately 1.5 times less than the actual value.

- the influx of the radiant heat arriving at the extended perimeter areas of specimens. With expansion factors (i.e., the difference between fully expanded coating thickness and pre-activated coating thickness, divided by pre-activated coating thickness) in a general range of 5 to 62 , identified in previous studies $[3,10]$, the surface area of fully extended perimeter surfaces easily exceeds that of the top surface on which heat is mainly absorbed, which is normally $0.01 \mathrm{~m}^{2}$ in dimension. Under the changed exposure conditions, the quantity of the heat absorbed by the perimeter areas can comprise a noticeable portion of the total heat absorption.

These two aspects, in terms of non-consistent thermal loading, cannot be neglected in any investigation utilising the cone calorimetry into material samples undergoing moving boundaries and appreciable side exposures.

Difficulties in theoretically solving the issues of (i) the variation in thermal load on top surfaces and (ii) the inclusion of the neglected irradiance on side surfaces originate from the unique spatial configuration between the truncated cone shaped emitter and a rectangular recipient. From the viewpoint of radiation transfer, this arrangement provokes that both the distance between each infinitesimal area of the two domains and the angles between the 
distance lengthwise line and each elemental area's normal vector vary all over the exposed surfaces of the recipient. The built-in geometrical characteristics of this apparatus, therefore, result in non-uniform dispersions of irradiance all over the exposed surfaces. In relation to this nonlinear thermal loading, recent research has established that the irradiance on the top surface is not uniformly distributed outside the $50 \mathrm{~mm}^{2}$ central area, by physically measuring irradiances or numerically calculating geometric view factors [13-16]. However, theoretical derivations from the principles of thermal radiation have not been adequately demonstrated to resolve the thermal issues in relation to the moving boundaries and side exposures.

This work aims to clarify the exact quantities of irradiances produced in bench-scale cone calorimeter tests so as to improve the subsequent assessment process of intumescent-type samples tested with the instrument. This objective is achieved by

- algebraically deriving view factors using contour integration to find relations for factors (i) from plane element to interior of truncated cone in parallel configuration; (ii) from plane element to segment of interior of truncated cone in perpendicular configuration.

- verifying the numerical calculations by direct measurements and pertinent existing data sets $[13,14]$.

- analysing the resultant impacts of the phenomena of (i) the non-uniform irradiance dispersions and (ii) the non-consistent thermal loads, induced by specimens' moving boundaries and perimeter area exposures, on the quantification of heat absorption, by considering a general intumescent-type specimen.

Calculated view factors are mapped on coordinate plane grids and presented by graphical representations. A series of direct measurements of irradiance are conducted using a Schmidt-Boelter gauge (Medtherm GTW-10-32-485A) in cooperation with Korea Conformity Laboratories (KCL).

\section{Methodology}

Methods of evaluating view factors have evolved to improve the accuracy, algebraic simplification and computational efficiency in various fields of engineering [17-28]. While integrating differential-element view factors over finite areas, Hamilton and Morgan [17] realised algebraic complexities, and initiated the use of Stokes' theorem to simplify their eventual solution. Sparrow [18] was among the first to utilise this vector calculus-based theorem, employing it in plate systems as a basic mathematical tool. This mathematical approach, which is henceforth referred to as the contour integration method, was examined in a study [19] into its accuracy and computational efficiency in comparison to other results obtained by using conventional methods. His research demonstrated the superiority of the contour integration method in both aspects. Its accuracy was also studied in existing literature [20], which concluded that this method yields very accurate values with mathematically simpler implementation as compared to the conventional area integration method. These studies proved that the contour integration method is a very powerful tool in assessing view factors.

View factors for the particular geometry of the cone calorimeter have recently attracted much attention for the improvement of test maturity and wide applications. Wilson et al. [13] calculated view factors for the exposed top surface of specimens, employing a formula 
expressing an arrangement between a plane element and a parallel circular disk, tabulated in a catalogue of factors [21]. For the identical geometry, Gemaque et al. [14] derived equations using area integration. For disk/rectangle and rectangle in parallel and perpendicular configurations, which are similar to the present configurations of the cone calorimetry, Abishek et al. [22] derived formulae using contour integration, demonstrating further detailed processes of derivation. In these previous studies, however, either little information was provided as to how the algebraic complexities of double (or quadruple) integrations of differential-element view factors were solved, or it was still non-transferable to finding the factor between segment of interior of truncated cone and perimeter surface in perpendicular configuration. For these reasons, the original Sparrow approach is the method of choice used for both of the geometric relations in this work.

\section{Theory}

\subsection{Theoretical relations between irradiance and view factor}

The irradiance diffusely emitted by the conical heater that arrives at each infinitesimal area on specimens $\left(\dot{q}_{d s}^{v \prime}\right)$ is quantified by two magnitudes, which are the radiant power emitted by the heat source per unit area $\left(\dot{q}_{\text {emit,haster }}^{\prime \prime}\right)$ and the view factor between the two domains $\left(F_{d s-h e a t e r}\right)$. Drawing upon an energy balance equation, the theoretical relations between the three key quantities can be defined using the reciprocity relation for view factors [21,23], as follows:

$$
\begin{gathered}
\dot{q}_{d s}^{\prime \prime}=\frac{d F_{\text {heater-ds }}\left(A_{\text {heater }} \dot{q}_{\text {emit,heater }}^{\prime \prime}\right)}{d A_{s}}=F_{d s-\text { heater }} \dot{q}_{\text {emit }, \text { heater }}^{\prime \prime} \\
\dot{q}_{\text {emit, heater }}^{\prime \prime}=\frac{\dot{q}_{a b s, g}^{\prime \prime}}{\alpha_{g} F_{g-h e a t e r}}
\end{gathered}
$$

The dash in the subscript of $F$ implies "to".

To obtain the irradiance $\dot{q}_{d g}^{\prime \prime}$ using Eqs. (1) and (1a), four parameters which are $\alpha_{g}, \dot{q}_{a b s, g}^{\prime \prime}$, $F_{g \text {-heater }}$ and $F_{d s-h e a t e r}$ must be known. The absorptivity of the Schmidt-Boelter gauge $\left(\alpha_{g}\right)$ is close to unity due to the condition of its measuring surface which is coated with a matt black finish. The second parameter $\left(\dot{q}_{a b s g}^{n \prime}\right)$ is typically obtained by direct measurements using the heat flux meter in calibration stages. The view factor from the gauge to the heater $\left(F_{\text {g-heater }}\right)$ can be obtained as a consequence of successful derivations of the last parameter $F_{d s-h e a t e r}$. Hence, this work focuses on deriving an elemental view factor from a diffuse differential area of specimens' exposed surfaces to the interior of the conical heater $\left(F_{d s-h e a t e r}\right)$ to clarify the exact quantities of irradiance produced in cone calorimeter tests.

\subsection{View factor for the top surface of specimens}

The spatial configuration between the top surface of specimens $\left(A_{l}\right)$ and the inclined/curved area of the conical heater $\left(A_{3}\right)$, as detailed in Fig. 2, caused difficulties in deriving a differential-element view factor from $d A_{1}$ to $A_{3}\left(F_{d A 1-A 3}\right)$. Its derivation can be simplified by considering a substitute for this uncommon arrangement based on the principle of energy 
conservation [21]. In other words, the solid angle positioned on $d A_{1}$ and subtended when viewing $A_{3}$ should correspond to the difference between the solid angle subtended when viewing $A_{2}$ (the yellow coloured cone based to $d A_{1}$ ) and that when viewing $A_{4}$ (the blue coloured cone). Hence, the diffuse view factor $F_{d A 1-A 3}$ was defined with the set of the surface areas in parallel configuration $\left(d A_{1}, A_{2}\right.$ and $\left.A_{4}\right)$, as follows:

$$
F_{d A 1-A 3}=F_{d A 1-A 2}-F_{d A 1-A 4}
$$

Despite this simplification, there were still algebraic complexities in evaluating $F_{d A 1-A 3}$ due to the existence of double (area) integrals shown in Eq. (3); the pertinent geometric relation between the parallel areas is detailed in Fig. 3a.

$$
\begin{aligned}
F_{d 11-A 3} & =\int_{\rho=0}^{r_{2}} \int_{\varphi=0}^{2 \pi} \frac{h_{2}^{2} \rho}{\pi\left\{p^{2}+\rho^{2}+h_{2}^{2}-2 p \rho \cos (\theta-\varphi)\right\}^{2}} d \varphi d \rho \\
& -\int_{\rho=0}^{r_{4}} \int_{\varphi=0}^{2 \pi} \frac{h_{4}{ }^{2} \rho}{\pi\left\{p^{2}+\rho^{2}+h_{4}{ }^{2}-2 p \rho \cos (\theta-\varphi)\right\}^{2}} d \varphi d \rho
\end{aligned}
$$

In vector calculus, Stokes' theorem is capable of assisting area integrals in transforming into contour integrals by utilising its mathematical equilibrium. This equilibrium indicates that a vector normal to an infinitesimal area is mathematically equivalent to the combination of the contour vectors surrounding this area. Based on this principle, Sparrow [18] elaborated upon mathematical transformations of area integrals in conventional formulae into contour integrals, and proposed a general equation for view factors between an elemental area and a finite area, as expressed in Eq. (4).

$$
\begin{aligned}
F_{d 41-A 2} & =\iint_{A_{2}}(\vec{\nabla} \times \vec{F}) \cdot d \vec{A}_{2}=\oint_{C_{2}} \vec{F} \cdot d \vec{\ell} \\
& =\ell_{1} \oint_{C_{2}} \frac{\left(z_{2}-z_{1}\right) d y_{2}-\left(y_{2}-y_{1}\right) d z_{2}}{2 \pi s_{2}^{2}} \\
& +m_{1} \oint_{C_{2}} \frac{\left(x_{2}-x_{1}\right) d z_{2}-\left(z_{2}-z_{1}\right) d x_{2}}{2 \pi s_{2}^{2}} \\
& +n_{1} \oint_{C_{2}} \frac{\left(y_{2}-y_{1}\right) d x_{2}-\left(x_{2}-x_{1}\right) d y_{2}}{2 \pi s_{2}^{2}}
\end{aligned}
$$

$s_{2}$ is an abbreviation for

$$
s_{2}^{2}=p^{2}+r_{2}^{2}+h_{2}^{2}-2 p r_{2} \cos (\theta-\varphi)
$$

To perform the mathematical transformation process, the areal element-element system, shown in Fig. 3a, was substituted with an areal element-contour system between $d A_{1}$ and a general point $P_{2}$ (or $P_{4}$ ) along the closed contour $C_{2}$ (or $C_{4}$ ), as shown in Fig. 3b. It is noted that the unit normal vectors $\vec{n}_{1}$ and $\vec{r}_{2}$ (or $\vec{n}_{4}$ ) denote the directions which the corresponding surfaces face; the vector $\vec{n}_{1}$ indicates the orientation of $A_{1}$, while $\vec{n}_{2}$ (or $\vec{n}_{4}$ ) represents the direction of the closed path $C_{2}$ (or $C_{4}$ ) in the evaluation of the view factor formulae; the contour-path direction decides the sine conventions of outcomes when integrands of equations are taken along the contour $C_{2}$ (or $C_{4}$ ).

The elemental view factor, $F_{d A 1-A 3}$, was derived from Eq. (4). The spatial positions of $d A_{1}, P_{2}$ and $P_{4}$ were defined in the element-contour system as $d A_{1}:\left(x_{1}, y_{1}, z_{1}\right)=(p \cos \theta, p \sin \theta, 0), P_{2}$ : $\left(x_{2}, y_{2}, z_{2}\right)=\left(r_{2} \cos \varphi, r_{2} \sin \varphi, h_{2}\right)$ and $P_{4}:\left(x_{4}, y_{4}, z_{4}\right)=\left(r_{4} \cos \phi, r_{4} \sin \phi, h_{4}\right)$. Since $d A_{1}$ was parallel 
to the $x-y$ plane and its normal vector faced upward, its direct cosines were determined as $\ell_{l}=0$, $m_{l}=0$ and $n_{l}= \pm 1$. These direct cosines were superimposed, which resulted in having the first and second terms of integrands in Eq. (4) removed. The downward-facing $A_{2}$ (or $A_{4}$ ), which was visible from $d A_{1}$, determined the direction of the closed path $C_{2}$ (or $C_{4}$ ), being described in the clockwise sense when viewed from above. Consequently, a contour integration-based formula for $F_{d A 1-A 3}$ was derived as follows.

$$
F_{d A 1-A 3}=\frac{1}{\pi}\left[\int_{\pi}^{0} \frac{p r_{2} \cos \varphi-r_{2}^{2}}{s_{2}^{2}} d \varphi-\int_{\pi}^{0} \frac{p r_{4} \cos \phi-r_{4}^{2}}{s_{4}^{2}} d \phi\right]
$$

The equation demonstrates a mathematically simpler execution as compared to Eq. (3) which is based on the conventional area integration method.

\subsection{View factors for the side surfaces of specimens}

The quantity of radiant heat transmitted from the conical heater to specimens' side surfaces is also determined by the two magnitudes, $\dot{q}_{\text {emit } h \text { atr }}^{\prime \prime}$ and $F_{d s-h e a t e r}$, as explained in Eqs. (1) and (1a). Unlike in the previous case for irradiance on the top surface, a portion of the total radiant power (in units of Watts) is involved only in the quantification of irradiance on the sides, as illustrated in Fig. 4. The yellow coloured segment, $A_{5}$, represents a surface area of the heater where viewed from an infinitesimal area of the perimeter surface $\left(d A_{l}\right)$, which indicates the actual heat source radiating to one of the side surfaces. It is noted that the mutual visibility varies with the horizontal distance of the vertical area $A_{l}$ from the origin (i.e., $p$ ), which represents the half length of given test samples. Due to the shape of the truncated cone, the variable visibilities between $A_{5}$ and $d A_{1}$ need to be categorised into two cases according to the range of $p$ as (i) at $r_{4} \leq p<r_{2}$ and (ii) at $p<r_{4}$, as detailed in Figs. $4 \mathrm{a}$ and $4 \mathrm{~b}$ respectively, for the standardised bench-scaled instrument which is $r_{2}=80 \mathrm{~mm}, r_{4}=40 \mathrm{~mm}$ and $H=65 \mathrm{~mm}$ in dimensions $[1,12]$. It is also noted that, on condition that the square top surface of samples is equal to or greater than $160 \mathrm{~mm}^{2}$ (i.e., $p \geq r_{2}$ ), irradiances on their sides are no longer an appreciable consideration as these areas are not visible from the conical heater.

In relation to the evaluation of the view factor from $d A_{1}$ to $A_{5}$, the solid angle located on $d A_{1}$ and subtended by $A_{5}$ should correspond to either (i) the solid angle subtended by the projected area of $A_{5}$ onto the $x-y$ plane (i.e., $A_{6}$ ) at $r_{4} \leq p<r_{2}$, or (ii) the difference between the solid angle subtended when viewing $A_{6}$ and that when viewing $A_{7}$ at $p<r_{4}$, based on the principle of energy conservation. This facilitated the process of view factor derivations with the set of the areas in perpendicular configuration $\left(d A_{1}, A_{6}\right.$ and $\left.A_{7}\right)$, which gave the elemental view factor $F_{d A 1-A 5}$ as $F_{d A 1-A 6}$ at $r_{4} \leq p<r_{2}$, or as the difference between $F_{d A 1-A 6}$ and $F_{d A 1-A 7}$ at $p<r_{4}$.

The mathematical derivation of $F_{d A 1-A 5}$ originated from Eq. (4). In the same manner as shown in the previous substitution process based on Stokes' theorem, the areal element-element system between $d A_{1}$ and $A_{6}$ ( or $A_{7}$ ) was replaced with an element-contour system between $d A_{1}$ and a general point $P$ on the closed boundary of $A_{6}$ (or $A_{7}$ ), as detailed in Fig. 5. It is noted that the contour of $A_{6}$ needs to be divided into a straight line path $\left(C_{\ell}\right)$ and an arc path $\left(C_{a}\right)$ to evaluate the integrals over the different paths. The intervals of integrations for the separated contours were limited from $-r_{2} \sin \omega_{0}$ to $r_{2} \sin \omega_{0}$ and from $-\omega_{0}$ to $\omega_{0}$ respectively, owing to their dependence on the dimension of given specimens. The coordinates 
of $d A_{1}, P_{\alpha, 2}$ and $P_{\ell, 2}$ were defined as $d A_{1}:\left(x_{1}, y_{1}, z_{1}\right)=(p, q,-t), P_{\alpha, 2}:\left(x_{\alpha, 2}, y_{\alpha, 2}, z_{\alpha, 2}\right)=\left(r_{2} \cos \varphi\right.$, $\left.r_{2} \sin \varphi, h_{2}\right)$ and $P_{\ell, 2}:\left(x_{\ell, 2}, y_{\ell, 2}, z_{\ell, 2}\right)=\left(p, y_{\ell, 2}, h_{2}\right)$. Considering the orientation of $d A_{1}$, its direct cosines were determined as $\ell_{1}= \pm 1, m_{l}=0$ and $n_{l}=0$, and superimposed. This resulted in the second and third terms in Eq. (4) vanishing. Consequently, a contour integration-based formula for $F_{d A 1-A 5}$ was defined as follows, however this is valid only at $r_{4} \leq p<r_{2}$.

$$
\begin{aligned}
F_{d A 1-A 5} & =\oint_{C_{a, 2}}\left[\frac{1}{2 \pi} \frac{\left(z_{a, 2}-z_{1}\right) d y_{a, 2}-\left(y_{a, 2}-y_{1}\right) d z_{a, 2}}{s_{a, 2}^{2}}\right] \\
& +\oint_{C_{\ell, 2}}\left[\frac{1}{2 \pi} \frac{\left(z_{\ell, 2}-z_{1}\right) d y_{\ell, 2}-\left(y_{\ell, 2}-y_{1}\right) d z_{\ell, 2}}{s_{\ell, 2}^{2}}\right] \\
& =\frac{1}{2 \pi}\left[\int_{\varphi_{0}}^{-\varphi_{0}} \frac{\left(h_{2}+t\right)\left(r_{2} \cos \varphi\right)}{s_{a, 2}^{2}} d \varphi+\int_{-r_{2} \sin \varphi_{0}}^{r_{2} \sin \varphi_{0}} \frac{\left(h_{2}+t\right)}{s_{\ell, 2}^{2}} d y_{\ell, 2}\right]
\end{aligned}
$$

$s_{a, 2}$ and $\mathrm{s}_{\ell, 2}$ are abbreviations for

$$
\begin{aligned}
& {s_{a, 2}}^{2}=p^{2}+q^{2}+r_{2}^{2}+h_{2}^{2}+t^{2}+2 h_{2} t-2 r_{2}(p \cos \varphi+q \sin \varphi) \\
& {s_{\ell, 2}}^{2}=q^{2}+y_{\ell, 2}{ }^{2}+h_{2}^{2}+t^{2}+2\left(h_{2} t-y_{\ell, 2} q\right)
\end{aligned}
$$

When a specimen with a top surface area smaller than $80 \mathrm{~mm}^{2}$ is subjected to the apparatus (i.e., $p<r_{4}$ ), an additional algebraic term is required to explain the portion of the illumination arriving at the upper circular segment $A_{7}$, as shown in Fig. $4 \mathrm{~b}$. Hence, for this case, the formula for $F_{d A 1-A 5}$ was defined as follows:

$$
\begin{aligned}
F_{d A 1-A 5} & =\frac{1}{2 \pi}\left[\int_{\varphi_{0}}^{-\varphi_{0}} \frac{\left(h_{2}+t\right)\left(r_{2} \cos \varphi\right)}{s_{a, 2}^{2}} d \varphi+\int_{-r_{2} \sin \varphi_{0}}^{r_{2} \sin \varphi_{0}} \frac{\left(h_{2}+t\right)}{s_{\ell, 2}^{2}} d y_{\ell, 2}\right] \\
& -\frac{1}{2 \pi}\left[\int_{\phi_{0}}^{-\phi_{0}} \frac{\left(h_{4}+t\right)\left(r_{4} \cos \phi\right)}{s_{a, 4}^{2}} d \phi+\int_{-r_{4} \sin \phi_{0}}^{r_{4} \sin \phi_{0}} \frac{\left(h_{4}+t\right)}{s_{\ell, 4}^{2}} d y_{\ell, 4}\right]
\end{aligned}
$$

where $P_{a, 4}:\left(r_{4} \cos \phi, r_{4} \sin \phi, h_{4}\right), P_{\ell, 4}:\left(p, y_{\ell, 4}, h_{4}\right) . \mathrm{s}_{a, 4}$ and $\mathrm{s}_{\ell, 4}$ are abbreviations for

$$
\begin{aligned}
& s_{a, 4}{ }^{2}=p^{2}+q^{2}+r_{4}^{2}+h_{4}^{2}+t^{2}+2 h_{4} t-2 r_{4}(p \cos \phi+q \sin \phi) \\
& s_{\ell, 4}{ }^{2}=q^{2}+y_{\ell, 4}{ }^{2}+h_{4}^{2}+t^{2}+2\left(h_{4} t-y_{\ell, 4} q\right)
\end{aligned}
$$

\section{Experimental details}

The physical measurements conducted by KCL were intended to collect data sets of irradiance capable of verifying the numerical predictions obtained by using Eqs. (1) and (5-7). With respect to irradiance on specimens' top surfaces, a total of seventeen positions in a radial pattern based around the central point of specimens were selected, as detailed in Fig. 6a. This radial pattern design was based on an understanding that the incident of radiant flux at any position on the top surface is dependent on the horizontal distance from the centre due to the shape of the truncated cone. As regards irradiance on samples' side surfaces, its quantity was measured by rotating the measuring face of the heat flux meter through 90 degrees, as shown in Fig. 6b. A total of eight positions, identically distanced $25 \mathrm{~mm}$ and $50 \mathrm{~mm}$ from the centre but facing outward in different directions, were chosen. Figs. 7a and $7 \mathrm{~b}$ show the photographs demonstrating the two types of experimental setups, respectively. 
The multiple positions were designed to provide for experimental uncertainties, which could be possibly generated by an angular or functional imperfection of the heating element. The two sets of measuring positions were regulated at $h=15 \mathrm{~mm}, 25 \mathrm{~mm}, 35 \mathrm{~mm}$ and $45 \mathrm{~mm}$ underneath the conical heater. These specifications gave the particulars of irradiance dispersions in the z-direction. A radiant heat flux of $50 \mathrm{~kW} / \mathrm{m}^{2}$ was initially regulated at the central position $25 \mathrm{~mm}$ underneath the heater. The constant radiant power (in units of Watts) of the heater gained when the monitor of the heat flux probe displays this quantity $\left(50 \mathrm{~kW} / \mathrm{m}^{2}\right)$ was served as a reference point in all the measurements.

\section{Results and discussion}

View factor maps are created on coordinate plane grids, which are horizontally and vertically oriented, to demonstrate the nonlinear view factor dispersions over the three-dimensional physical space underneath the conical heater. With these data sets, irradiances are predicted by using Eq. (1a). Subsequently, variations in heat absorption according to the levels of geometric progressions of the boundaries of a general intumescent-type specimen are explored. Prior to conducting this process, the reliability and accuracy of the numerical calculations of view factors using the contour integration method are verified by the existing literature $[13,14]$ and the direct measurements.

\subsection{Validations}

In prior studies $[13,14]$, either the view factor $F_{d A 1-A 3}$ or $F_{A 3-A 1}$ was calculated for the geometric configuration between the conical heater $\left(A_{3}\right)$ and the top surface of $100 \mathrm{~mm}^{2}\left(A_{1}\right)$. It is important to mention that $F_{A 3-A I}$ is obtained by evaluating the integral of $d F_{A 3-d A I}$ over $A_{1}$, using the reciprocity relation between $d F_{A 3-d A 1}$ and $F_{d A 1-A 3}$, as explained in Eq. (1). Table 1 shows the values of $F_{d A 1-A 3}$ and $F_{A 3-A l}$ when the top surface is positioned at intervals of $5 \mathrm{~mm}$ vertical distance from the heater, from $h=15 \mathrm{~mm}$ to $h=35 \mathrm{~mm}$. This comparative table demonstrates the credibility of the present calculations, up to four significant digits.

The direct measurements further validated the assessment of view factors conducted in this work. The markers in Figs. 8a and $8 \mathrm{~b}$ indicate the respective data sets of irradiances measured using the Schmidt-Boelter gauge under the referenced heating condition introduced in Section 3. The different style lines superimposed on the sets of markers denote the numerical predictions, which demonstrate the variations in irradiances with the changes in $h$ and $p$. It can be observed from Fig. 8a that the maximum difference between numerical predictions and physical measurements was 4.14 per cent at $h=15 \mathrm{~mm}$ and $p=50 \mathrm{~mm}$. Although a slightly greater discrepancy was identified in Fig. $8 \mathrm{~b}$ when $h=15 \mathrm{~mm}$ and $p=50 \mathrm{~mm}$, overall the predictions were in agreement with the measurement data.

With respect to uncertainty analyses on the heat flux measurements, three influential sources, which are (i) random error by measurements, (ii) resolution error by the gauge indication, and (iii) correction error by the gauge calibration, were taken into account. The standard uncertainties $(u)$ for the respective sources related were estimated based on the uncertainty budget listed in Table 2, showing the components' evaluation type, probability distribution form, unit, and relevant details. A set of combined standard uncertainties $\left(u_{c}\right)$ were, 
then, calculated using the estimated data of $u$, by adopting summation in quadrature for addition. Individual effective degree of freedom per $u_{c}$ was determined using Welch-Satterthwaite equation to achieve each coverage factor $(k)$. Then a set of expanded standard uncertainties $(U)$ was acquired by multiplying $u_{c}$ with $k$. Table 3 shows the resultant sets of the average irradiances, physically measured at different $h$ and $p$, with $U$ on the basis of $k$.

\subsection{View factor mapping on horizontally oriented coordinate plane grids}

To produce view factor maps, a local Cartesian coordinate system composed of $x$-, $y$-, and $z$-axes was introduced. With the apparatus in the standardised dimension, the view factor mapping was performed within a volume range of $160 \mathrm{~mm} \times 160 \mathrm{~mm} \times 50 \mathrm{~mm}$, as illustrated in Fig. 9. The origin of this coordinate system $(O)$ was located at the centre of and $50 \mathrm{~mm}$ underneath the conical heater. The shaded horizontal and vertical planes represent the coordinate plane grids on which irradiance was imposed and on which, thus, view factor maps were built. The normal vectors $\vec{n}_{1}$ and $\vec{n}_{2}$ of the planes indicate the directions that the corresponding surfaces face, facing upward and outward respectively. It is noted that the two different view factors, which are to predict irradiances on the upward- and outward-facing surfaces, are henceforth referred to as $F_{d A 1-A 3}$ and $F_{d A 1-A 5}$ respectively, as used in Eqs. (5-7).

Fig. 10 shows the contour maps of $F_{d A 1-A 3}$ when $z=0 \mathrm{~mm}$ and $25 \mathrm{~mm}$. With respect to the dispersion of $F_{d A 1-A 3}$ over the plane with $\vec{r}_{1}$, overall it was in radial patterns with the z-axis as the centre. It was found that the view factor decreased as the horizontal distance of measuring points from the centre (i.e., $p=\sqrt{x^{2}+y^{2}}$ ) increased. In relation to the intensification of irradiance, as claimed in Introduction section, it was demonstrated that the overall view factors obtained when $z=25 \mathrm{~mm}$ were greater than those at $z=0 \mathrm{~mm}$. This indicates that the movement of specimens' top boundaries, toward the heater with a constant radiant power, certainly intensifies the radiant heat flux imposed on the top surface.

\subsection{View factor mapping on vertically oriented coordinate plane grid}

Figs. $11 \mathrm{a}$ and $11 \mathrm{~b}$ illustrate the $F_{d A 1-A 5}$ variations as functions of the $y$-and $z$-coordinates of the measuring point when the $y-z$ planes with $\vec{n}_{2}$ are horizontally $25 \mathrm{~mm}$ and $50 \mathrm{~mm}$ from the centre as described in the diagrams on the right. Overall, appreciable variations in $F_{d A 1-A 5}$ were observed in both the $y$ - and $z$-directions. Its variations in the $y$-direction can be more clearly understood from Figs. 12a and 12b; as the variations have a line of symmetry through $z$-axis, the outcomes for positive $y$-coordinates were expressed only. The solid lines with different marks indicate the reduction ratios (i.e., $F_{d A 1-A 5}$ at variable $y$, divided by $F_{d A 1-A 5}$ at $y=0 \mathrm{~mm}$ ) in percentage at corresponding z-coordinates, as described in the diagrams accompanying Figs. $12 \mathrm{a}$ and $12 \mathrm{~b}$. In a specific case of square specimens' top surfaces with a typical size of $100 \mathrm{~mm}$ x $100 \mathrm{~mm}$ (i.e., $x=y=50 \mathrm{~mm}$ ), $F_{d A 1-A 5}$ could be reduced by up to 55.7 per cent of its maximum at the edge (when $y=50 \mathrm{~mm}$ ), as detailed in Fig. 12b. These results demonstrate that the irradiance dispersions over specimens' side surfaces are highly nonlinear, which cannot be neglected in quantifying the heat absorbed by the side surfaces exposed to the conical heater.

To observe the $F_{d A 1-A 5}$ variations in the z-coordinate, the data sets obtained from the view factor maps were normalised such that the values at $z=0 \mathrm{~mm}$ were equal to unity, as shown in 
Figs. $13 \mathrm{a}$ and $13 \mathrm{~b}$. It was found from Fig. $13 \mathrm{~b}$ that $F_{d A 1-A 5}$ at $y=40 \mathrm{~mm}$ and $z=45 \mathrm{~mm}$ was approximately twelve times more than that at $z=0 \mathrm{~mm}$, which indicates that twelve times more irradiance is imposed on this position. It is noted that the exceptions observed when $y=80 \mathrm{~mm}$ were due to the unusual geometrical conditions of the incident angles and distances created by the configuration between the segmental inclined/curved surface of the heat source $\left(A_{5}\right.$ in Fig. 4) and the position at the edge of the vertical plane.

\subsection{Analysis of the resultant effects of non-uniform and non-consistent irradiances}

With the established view factor maps, the effect of the non-uniform irradiance dispersions over samples' top surfaces was quantitatively analysed by comparing two sub-models, each of which either includes or excludes the nonlinearity. It was assumed that the top surface was $100 \mathrm{~mm}$ x $100 \mathrm{~mm}$ in dimension, and its absorptivity was unity. Specimens' perimeter surfaces were not considered in this sub-analysis. Radiant energy absorbed by the horizontal plane was theoretically quantified by using Eq. (1a) under the referenced heating condition $\left(\dot{q}_{a b s, g}^{\prime \prime}=50 \mathrm{~kW} / \mathrm{m}^{2}\right.$ at the centre when $\left.h=25 \mathrm{~mm}\right)$. Fig. 14 shows the variations in the quantities of radiation absorption as a function of the $\mathrm{z}$-coordinate of the top surface, obtained by the two sub-models. It can be observed that the conventional method overestimated the heat absorption in the range between $z=0 \mathrm{~mm}$ and $z=30 \mathrm{~mm}$ while underestimated it in the range beyond $\mathrm{z}=30 \mathrm{~mm}$, as compared to the exact values. At $z=25 \mathrm{~mm}$, which is one of the most standard vertical distances of specimens from the cone baseplate in cone calorimeter tests, it resulted in a $0.016 \mathrm{~kW}$ overestimate. The dotted line with circular marks indicates discrepancy factor (i.e., the absolute difference between conventional value and exact value, divided by exact value) in percentage, which highlights the effect of the irradiance non-uniformity in cone calorimeter tests.

The effect of the non-consistent thermal loads, caused by specimens' moving boundaries and side exposures, on the overall heat absorption was also analysed, by considering a general intumescent-type sample with a top surface of $100 \mathrm{~mm} \times 100 \mathrm{~mm}$ under the top and side exposure conditions. It was assumed that this specimen was initially placed $50 \mathrm{~mm}$ underneath the conical heater with the referenced radiant power introduced in Section 3, and was systematically expanded up to the thickness of $45 \mathrm{~mm}$, as illustrated in Fig. 15a. Under the circumstances, the amounts of radiant heat absorbed by the top and side surfaces of the recipient were respectively calculated to identify each contribution, using Eq. (1a) and the view factor maps. In the calculations, the absorptivity of the exposed surfaces was assumed as unity.

Fig. 15b highlights this issue and shows its resultant data, in terms of the variations in the radiant heat absorbed by the top and side surfaces (in units of $\mathrm{kW}$ ) and their respective contributions as a function of the specimens' thickness progression (i.e., $z$ ). The sectors filled with grey solid and pattern (faint) colours in the bar charts indicate these quantities, while the solid and dotted lines refer to the total amounts obtained by the present and conventional methods, respectively. It is worth noting that, in the conventional approach, the total quantity has been approximated by direct measurements performed prior to the activation of intumescent-type materials, without taking into consideration the irradiance on the side surfaces. 
It was found that the difference in quantities between the conventional approximation and the present prediction became wider as the thickness progression developed. When the specimen was expanded beyond $30 \mathrm{~mm}$, the exact amount of heat absorption became more than twice as much as that approximated using the conventional method. With respect to respective contributions, the quantity of heat absorbed through the top surface gradually increased from $0.36 \mathrm{~kW}$ to $0.55 \mathrm{~kW}$ as the top surface spatially approached the conical heater. Concurrently with this rise, the amount of heat absorbed by the side surfaces also increased but more significantly. Its contribution even exceeded beyond the top surface's at $z=45 \mathrm{~mm}$, which is in close relation to the considerable increase in the corresponding side surface area up to $0.018 \mathrm{~m}^{2}$, as compared to the constant top surface area of $0.01 \mathrm{~m}^{2}$. However, at $z=25 \mathrm{~mm}$, when the surface area of the sides is equivalent to that of the top surface, its contribution accounted for 28.4 per cent of the total heat absorption, which proves that the top surface is still the primary area by which the majority of radiant heat is absorbed.

\section{Conclusions}

In fire tests, irradiance is a highly critical data to achieve a more complete understanding of heat-related characteristics of given materials. Since the cone calorimeter is capable of creating controlled heating environments, its application has been expanded to examining thermal behaviours and performances of intumescent-type fire retardant systems. In this expanded application, however, there still exist doubts over thermal boundaries of such specimens, due to their moving boundaries and appreciable side exposures in the course of tests. The conventional approach has not reflected the resultant non-consistent thermal loading during tests, which indicates (i) the intensification of irradiance on moving top boundaries and (ii) the influx of radiant heat on extended perimeter areas, owing to a lack of information on clear algebraic derivations of view factor for the spatial configuration of the bench-scaled cone calorimetry. Even through several studies on irradiance in the tests attempted to predict this magnitude using the view factor equations tabulated in the catalogue, and established the non-uniform irradiance dispersion on samples' top surfaces, these efforts proved unable to resolve the issue of the view factor derivation for the geometric relation between the truncate cone and the perimeter surface. Hence, this work set out to clarify the exact quantities of irradiances generated in the bench-scale cone calorimetry by calculating view factors using the contour integration method.

Two geometric relations (i) between plane element and interior of truncated cone in parallel configuration; (ii) between plane element and segment of interior of truncated cone in perpendicular configuration were investigated. The use of contour integration demonstrated clear mathematical implementations in solving double (area) integrations. The accuracy of the calculated view factors was validated through comparisons with the results, obtained from both existing literature and direct measurements, which showed good agreements up to four significant digits. It can be concluded that this method was highly compatible with the distinctive configuration between the conical heater and the side surfaces, as well as the top surface. Even though the view factors for the top surface could be calculated by using the existing catalogue of factors, the calculation of the factors for the side surfaces would not have been achieved if using these tabulated equations. 
The view factor maps created on both the horizontally and vertically oriented coordinate plane grids were designed to present graphical representations of irradiances on any position underneath the conical heater, based on a local Cartesian coordinate system. Consequently, the patterns and tendencies of view factor variations observed in these maps improved the understanding of nonlinear irradiance dispersions generated in cone calorimeter tests. Particularly, the application of these maps is highly recommended for any case which requires more detailed data of heat absorption to test specimens experiencing geometrical changes in the course of the cone calorimeter tests.

The influences of the phenomena of (i) the non-uniform irradiance dispersions and (ii) the non-consistent thermal loads were quantitatively recognised. It was found that the discrepancy between the exact calculation and the conventional approximation, each of which either includes or excludes the first phenomenon, was recorded up to 12.8 per cent of the exact value. A more significant deviation from the exact calculation was resulted when the second phenomenon was neglected as done by conventional approaches. It was demonstrated that this disagreement was induced by primarily the increase in the heat absorption through the perimeter areas, developed as they were extended, and by secondarily the rise in the heat absorption through the top surface, developed as it physically approached the heater. These aspects, therefore, should not be ignored in the quantification of heat absorption.

This integrated analysis of heat absorption, incorporating the assessment of both view factor and irradiance, can promote the understanding of the heat-related characteristics of various materials tested with the bench-scaled cone calorimetry.

\section{Acknowledgements}

This project is funded by Korea Evaluation Institute of Industrial Technology through the Technology Innovation Program (Award No. 10041239: Fundamental technologies for development of nanoclay dispersed intumescent polymer composite in application for built environment and automobiles). 


\section{REFERENCES}

[1] BS 476-15 (ISO 5660-1), Fire tests on building materials and structures - Part 15: Method for measuring the rate of heat release of products, British Standard (1993).

[2] M. Bartholmai, R. Schriever, B. Schartel, Influence of external heat flux and coating thickness on the thermal insulation properties of two different intumescent coatings using cone calorimeter and numerical analysis, Fire and Materials 27 (4) (2003) $151-162$.

[3] G.J. Griffin, A.D. Bicknell, T.J. Brown, Studies on the effect of atmospheric oxygen content on the thermal resistance of intumescent, fire-retardant coatings, Journal of Fire Sciences 23 (4) (2005) 303-328.

[4] Y. Wang, U. Göransson, G. Holmstedt, A. Omrane, A model for prediction of temperature in steel structure protected by intumescent coating, based on tests in the cone calorimeter, in: Proceedings of the Eighth International Symposium on Fire Safety Sciences, Beijing, 2005, pp. 235-246.

[5] A. Omrane, Y.C. Wang, U. Göransson, G. Holmstedt, M. Aldén, Intumescent coating surface temperature measurement in a cone calorimeter using laser-induced phosphorescence, Fire Safety Journal 42 (1) (2007) 68-74.

[6] J.F. Yuan, Y.C. Wang, Prediction of intumescent coating performance under cone calorimeter-A mathematical approach to performance based design, in: Proceedings of the Fifth International Conference on.Structures in Fire, Shanghai, 2008, pp. 713-724.

[7] J. Zhang, M.A. Delichatsios, Determination of the convective heat transfer coefficient in three-dimensional inverse heat conduction problems, Fire Safety Journal 44 (5) (2009) 681-690.

[8] L.M.R. Mesquita, P.A.G. Piloto, M.A.P. Vaz, T.M.G. Pinto, Decomposition of intumescent coatings: comparison between a numerical method and experimental results, Acta Polytechnica 49 (1) (2009) 60-65.

[9] Z. Han, A. Fina, G. Malucelli, G. Camino, Testing fire protective properties of intumescent coatings by in-line temperature measurements on a cone calorimeter, Progress in Organic Coatings 69 (4) (2010) 475-480.

[10] Y. Zhang, Y.C. Wang, C.G. Bailey, A.P. Taylor, Global modelling of fire protection performance of intumescent coating under different cone calorimeter heating conditions, Fire Safety Journal 50 (2012) 51-62.

[11] V. Babrauskas, Specimen heat fluxes for bench-scale heat release rate testing, Fire and Materials 19 (6) (1995) 243-252.

[12] V. Babrauskas, The cone calorimeter, in: SFPE Handbook of Fire Protection Engineering, SFPE \& NFPA, Massachusetts, 2002, pp. 3.63-3.81. 
[13] M.T. Wilson, B.Z. Dlugogorski, E.M. Kennedy, Uniformity of radiant heat fluxes in cone calorimeter, in: Proceedings of the Seventh International Symposium on Fire Safety Science, Worcester, 2003, pp. 815-826.

[14] M.J.A. Gemaque, F.S. Costa, View factors of samples tested in cone and cylinder calorimeters, ASME Journal of Heat Transfer 134 (9) (2012) 094503-1.

[15] B. Schartel, M. Bartholmai, U. Knoll, Some comments on the use of cone calorimeter data, Polymer Degradation and Stability 88 (3) (2005) 540-547.

[16] J. Lindholm, A. Brink, M. Hupa, Influence of decreased sample size on cone calorimeter results, Fire and Materials 36 (1) (2012) 63-73.

[17] D.C. Hamilton, W.R. Morgan, Radiant-interchange configuration factors, NACA, Washington, 1952, pp. 1-110.

[18] E.M. Sparrow, A new and simpler formulation for radiative angle factors, ASME Journal of Heat Transfer 85 (1963) 81-88.

[19] A.B. Shapiro, Computer implementation, accuracy, and timing of radiation view factor algorithms, ASME Journal of Heat Transfer 107 (3) (1985) 730-732.

[20] A. Ambirajan, S.P. Venkateshan, Accurate determination of diffuse view factors between planar surfaces, International Journal of Heat and Mass Transfer 36 (8) (1993) 2203-2208.

[21] R. Siegel, J.R. Howell, Thermal Radiation Heat Transfer, fourth ed., Taylor \& Francis, London, 2002.

[22] S. Abishek, K.S. Ramanujam, S.S. Katte, View factors between disk/rectangle and rectangle in parallel and perpendicular planes, Journal of Thermophysics and Heat Transfer 21 (1) (2007) 236-239.

[23] E.R.G. Eckert, R.M. Drake Jr., Heat and Mass Transfer, McGraw-Hill Book Company Inc., New York, 1959.

[24] A.J. Buschman Jr., C.M. Pittman, Configuration factors for exchange of radiant energy between axisymmetrical sections of cylinders, cones, and hemispheres and their bases, NASA, Washington, 1961, pp. 1-48.

[25] A. Feingold, Radiant-interchange configuration factors between various selected plane surfaces, Proceedings of the Royal Society A: Mathematical Physical and Engineering Science 292 (1428) (1966) 51-60.

[26] T.J. Chung, J.Y. Kim, Radiation view factors by finite element, ASME Journal of Heat Transfer 104 (4) (1982) 792-795.

[27] S. Lin, P.M. Lee, J.C.Y. Wang, W.L. Dai, Y.S. Lou, Radiant-interchange configuration factors between a disk and a segment of a parallel concentric disk, International Journal of Heat and Mass Transfer 29 (3) (1986) 501-503. 
[28] J.C.Y. Wang, S. Lin, Radiant-interchange configuration factors inside segments of frustum enclosures of right circular cones, International Communications in Heat and Mass Transfer 13 (4) (1986) 423-432. 


\section{TABLES}

Table 1

Comparisons of view factors obtained by previous literature $[13,14]$ and this work.

\begin{tabular}{ccccc}
\hline \multirow{2}{*}{${ }^{\mathrm{a}}$} & \multicolumn{2}{c}{$F_{d A 1-A 3}{ }^{\mathrm{b}}$} & \multicolumn{2}{c}{$F_{A 3-A 1}$} \\
\cline { 2 - 5 } & Ref. [13] & Present & Ref. [14] & Present \\
\hline 15 & 0.7660 & 0.7660 & 0.2731 & 0.2730 \\
20 & 0.7599 & 0.7599 & 0.2628 & 0.2627 \\
25 & 0.7461 & 0.7461 & 0.2509 & 0.2508 \\
30 & 0.7261 & 0.7261 & 0.2383 & 0.2382 \\
35 & 0.7014 & 0.7014 & 0.2254 & 0.2253 \\
\hline
\end{tabular}

a vertical distance of the specimens' top surface from the cone baseplate

${ }^{\mathrm{b}} d A_{l}$ is positioned at the centre of samples

Table 2

An uncertainty budget in relation to physical measurements shown in Figs. 8a and 8b.

Source of uncertainty Type $\begin{aligned} & \text { Probability } \\ & \text { distribution }\end{aligned}$ Units Remarks

\begin{tabular}{ccccc}
\hline $\begin{array}{c}\text { Measurement } \\
\text { repeatability }\end{array}$ & A & Normal & Rectangular $\quad \mathrm{kW} / \mathrm{m}^{2}$ & $\begin{array}{c}\text { The number of repeated } \\
\text { measurements }=12\end{array}$ \\
$\begin{array}{c}\text { Resolution } \\
\text { Reference standard } \\
\text { calibration }\end{array}$ & B & Normal & $\begin{array}{c}\text { Resolution power }=0.01 \mathrm{~kW} / \mathrm{m}^{2} \\
\text { with a confidence of } 95 \%\end{array}$ \\
\hline
\end{tabular}

Table 3

Uncertainty analysis on physical measurements shown in Figs. 8a and 8b.

\begin{tabular}{cccccc}
\hline \multirow{2}{*}{$h^{\mathrm{a}}$} & \multicolumn{4}{c}{ Average irradiance with expanded uncertainty $(U), \mathrm{kW} / \mathrm{m}^{2}$} \\
\cline { 2 - 6 } & \multicolumn{3}{c}{ upward-facing surface $^{\mathrm{c}}$} & outward-facing surface $^{\mathrm{d}}$ \\
\cline { 2 - 6 } & $p^{b}=0$ & $p=25$ & $p=50$ & $p=25$ & $p=50$ \\
\hline \multirow{2}{*}{15} & $53.00 \pm 1.41$, & $55.30 \pm 1.47$, & $57.90 \pm 1.45$, & $23.80 \pm 0.63$, & $14.50 \pm 0.46$ \\
& $k^{e}=1.98$ & $k=1.98$ & $k=1.96$ & $k=1.98$ & $k=2.06$ \\
25 & $51.60 \pm 1.27$, & $52.70 \pm 1.30$, & $49.90 \pm 1.28$, & $19.40 \pm 0.53$, & $9.80 \pm 0.32$ \\
& $k=1.96$ & $k=1.96$ & $k=1.97$ & $k=1.99$ & $k=2.09$ \\
35 & $49.50 \pm 1.22$, & $49.00 \pm 1.21$, & $45.10 \pm 1.12$, & $13.60 \pm 0.73$, & $5.00 \pm 0.32$ \\
& $k=1.96$ & $k=1.96$ & $k=1.96$ & $k=2.45$ & $k=2.57$ \\
45 & $45.80 \pm 1.23$, & $44.70 \pm 1.18$, & $38.70 \pm 1.02$, & $10.80 \pm 0.67$, & $3.40 \pm 0.27$ \\
& $k=1.98$ & $k=1.98$ & $k=1.98$ & $k=2.57$ & $k=2.78$ \\
\hline
\end{tabular}

\footnotetext{
${ }^{\mathrm{a}}$ vertical distance of the heat flux probe from the cone baseplate

${ }^{\mathrm{b}}$ horizontal distance of the heat flux probe from the cone axis

${ }^{\mathrm{c}}$ for the measurements shown in Fig. 8a

${ }^{d}$ for the measurements shown in Fig. $8 b$

e coverage factor, providing a level of confidence of approximately $95 \%$
} 


\section{FIGURES}

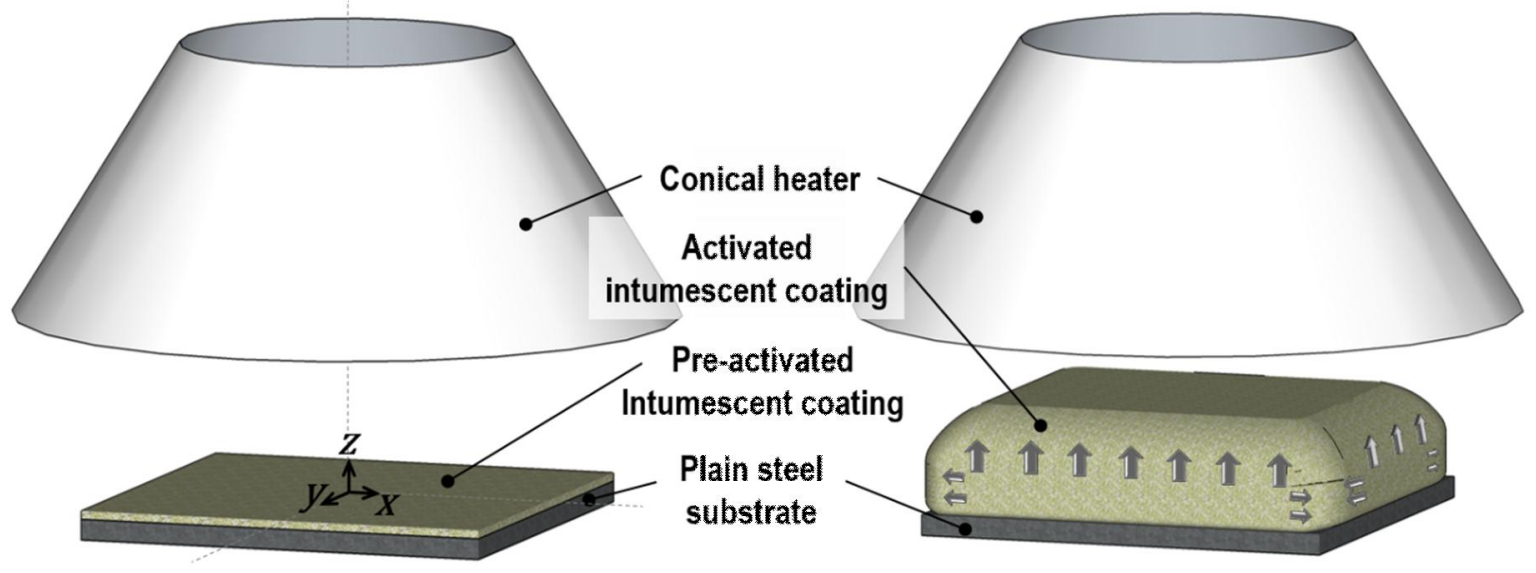

(a) Initial stage

(b) Intumescent stage

Fig. 1. Schematics of geometrical changes of intumescent systems occurring during cone calorimeter tests.

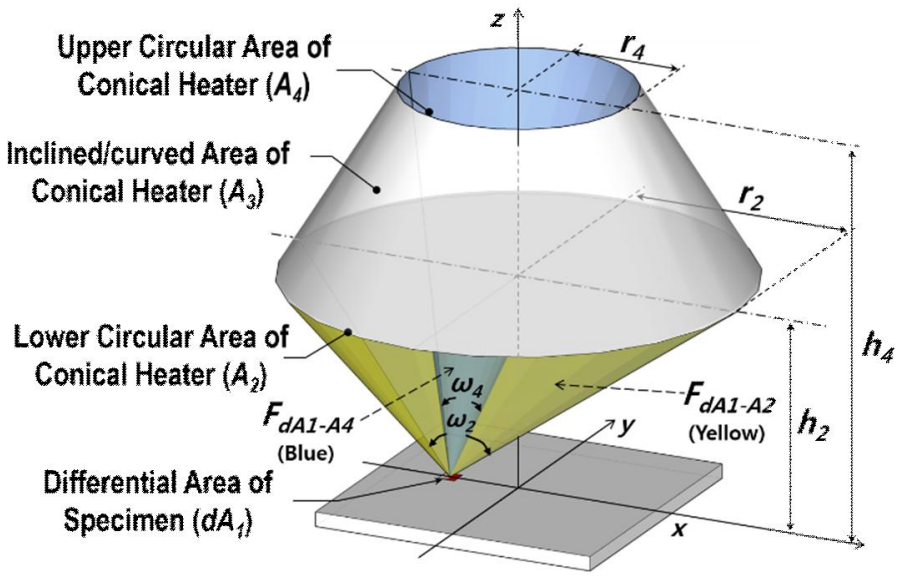

Fig. 2. A schematic of diffuse illumination transport from a differential area of the specimens' top surface to the conical heater. 


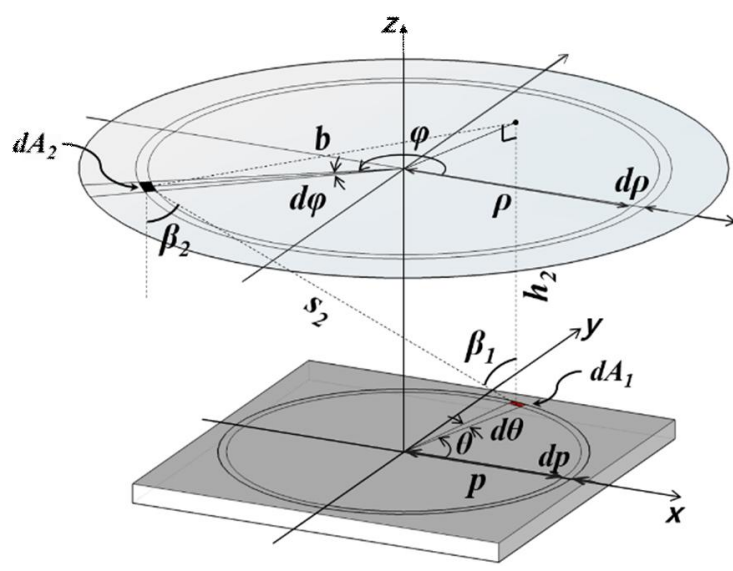

(a) Element-element system

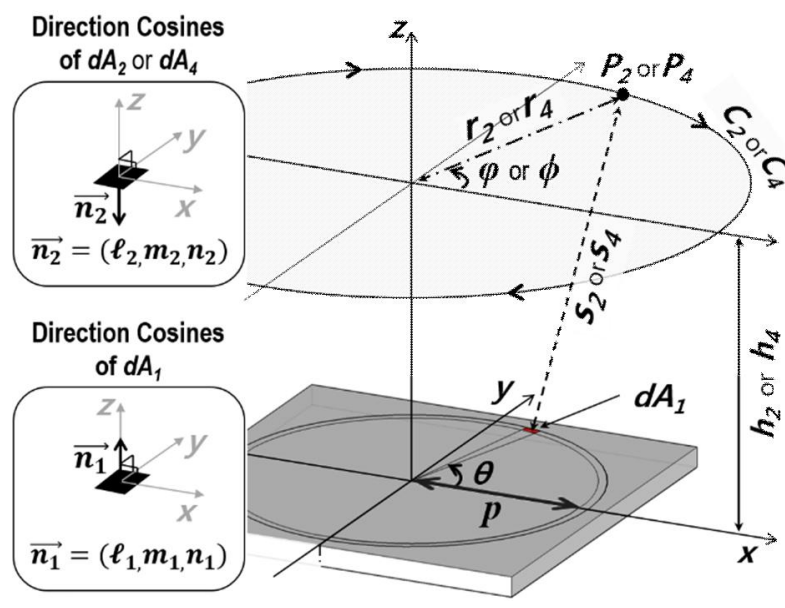

(b) Element-contour system

Fig. 3. Schematics of geometric systems for (a) the double integration method and (b) the contour integration method.

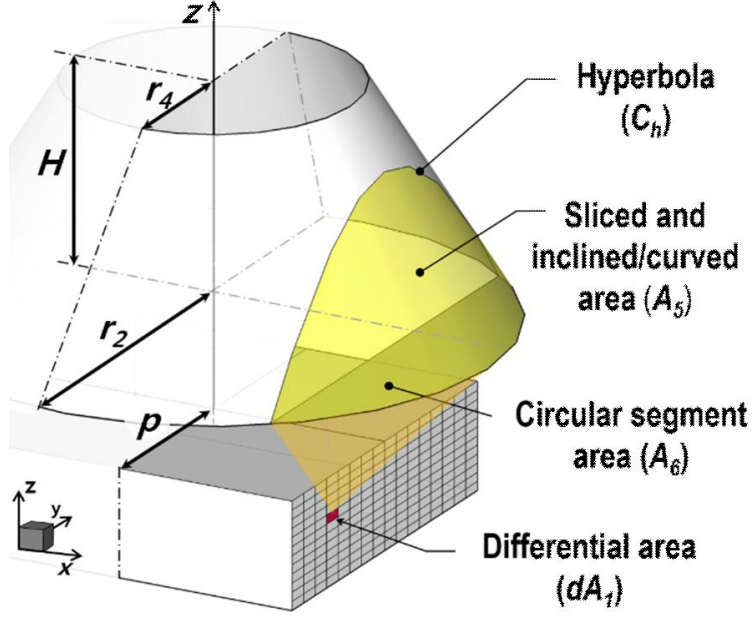

(a) $r_{4} \leq p<r_{2}$

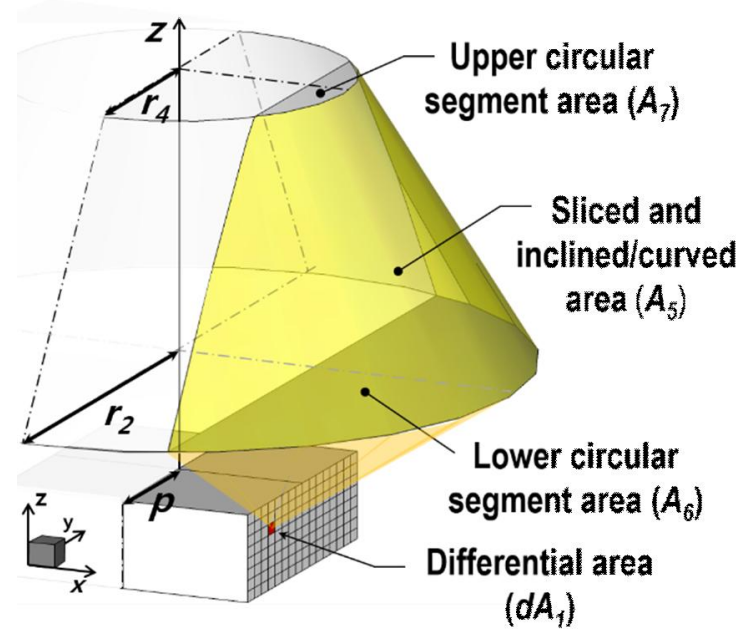

(b) $p<r_{4}$

Fig. 4. Schematics of diffuse illumination transport from a differential area of the specimens' side surface to the conical heater. 


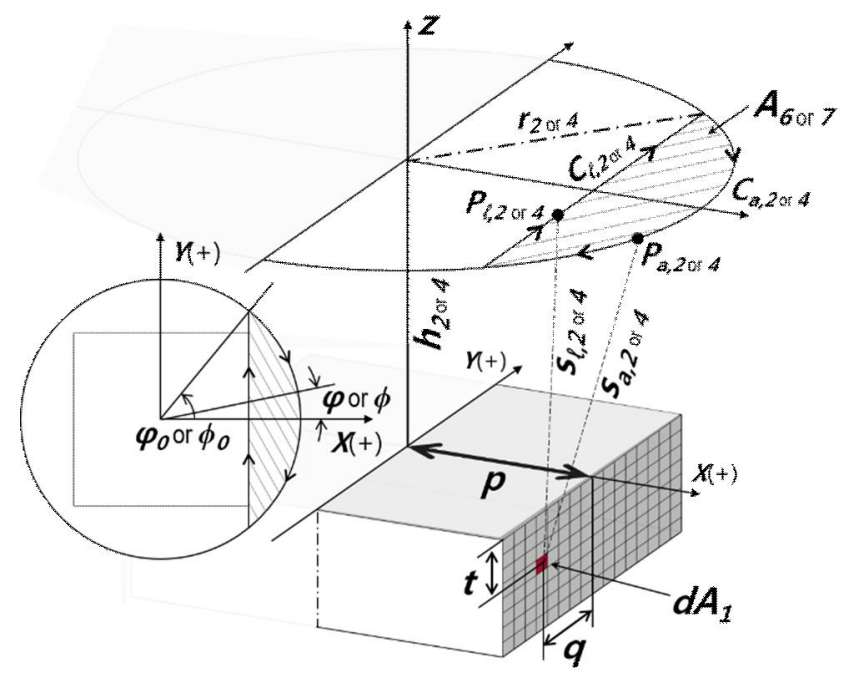

Fig. 5. A schematic of the element-contour system for configurations between the conical heater and the side surfaces of specimens.

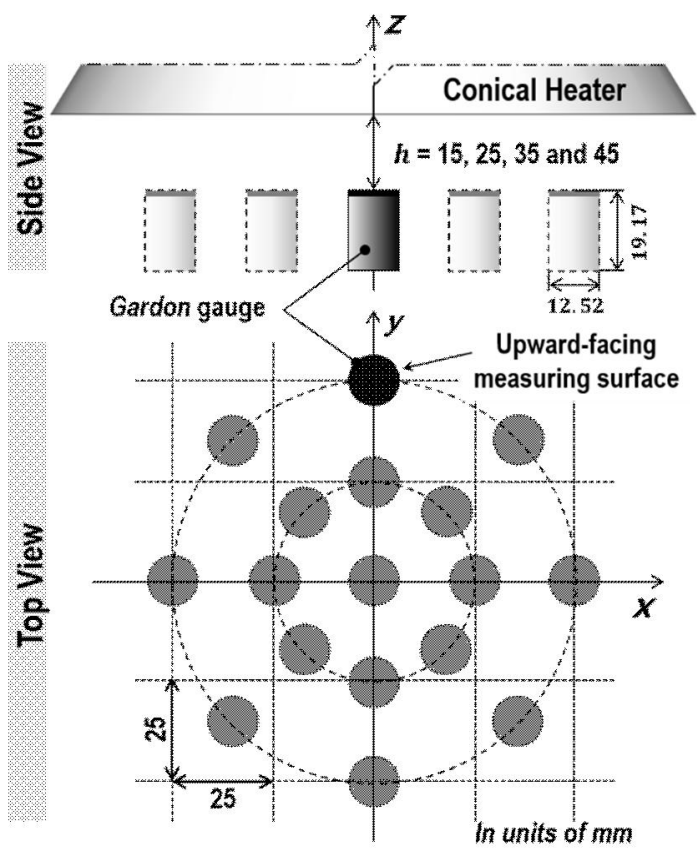

(a) upward-facing surface

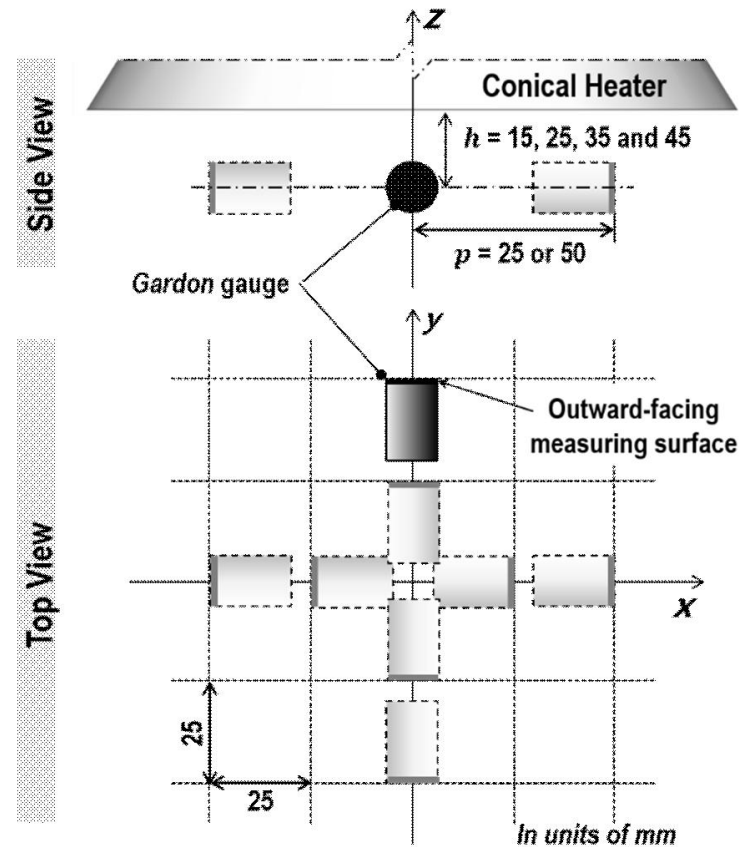

(b) outward-facing surface

Fig. 6. Schematics of direct measurement plans using a Schmidt-Boelter gauge. 


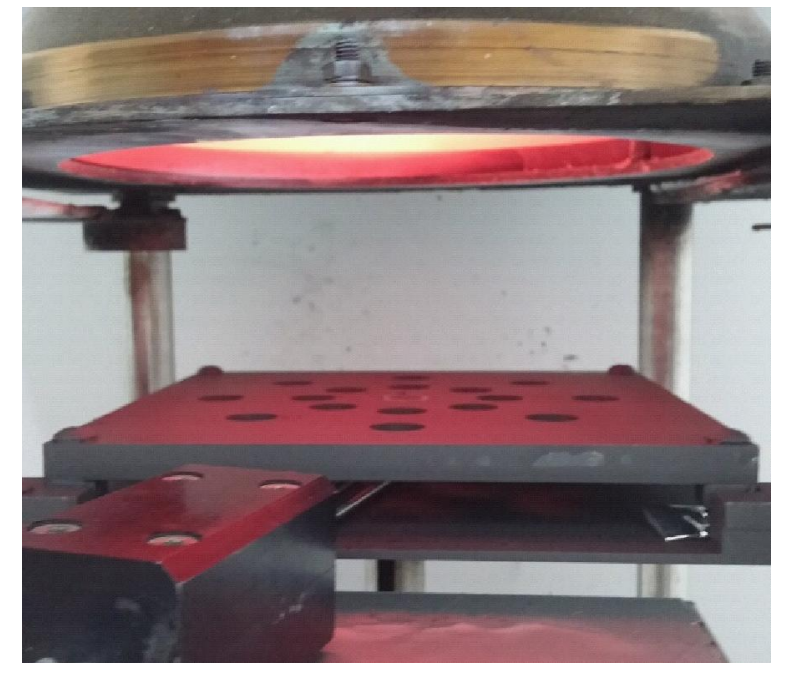

(a) upward-facing surface

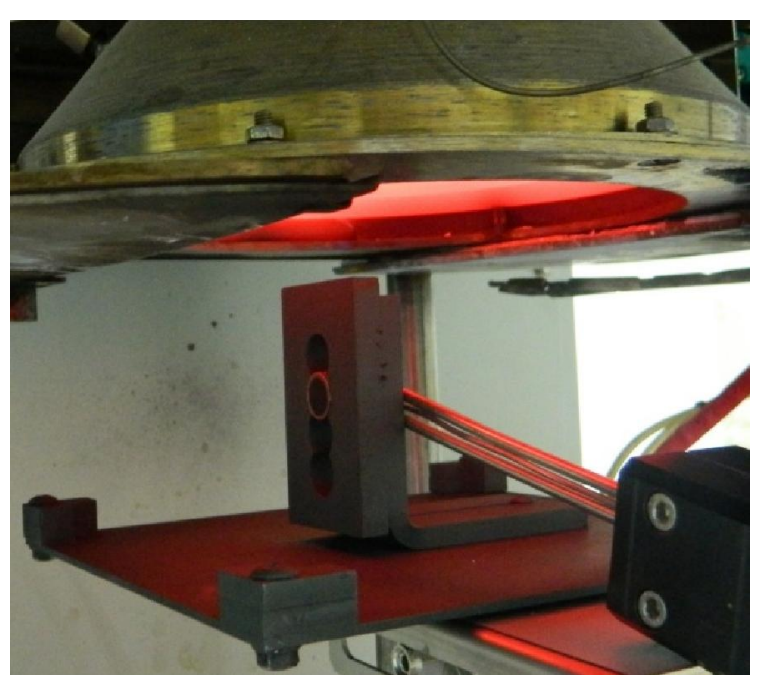

(b) outward-facing surface

Fig. 7. Photographic views of experimental setups.

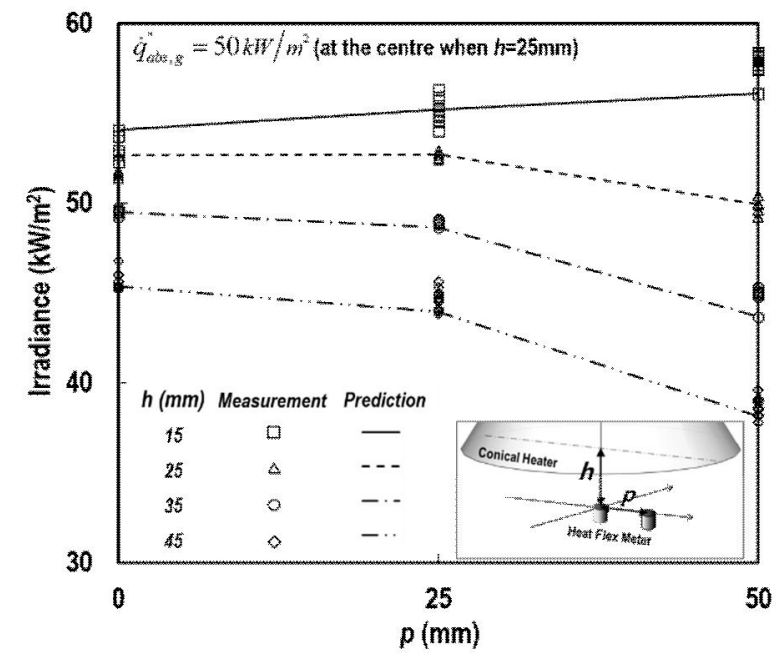

(a) upward-facing surface

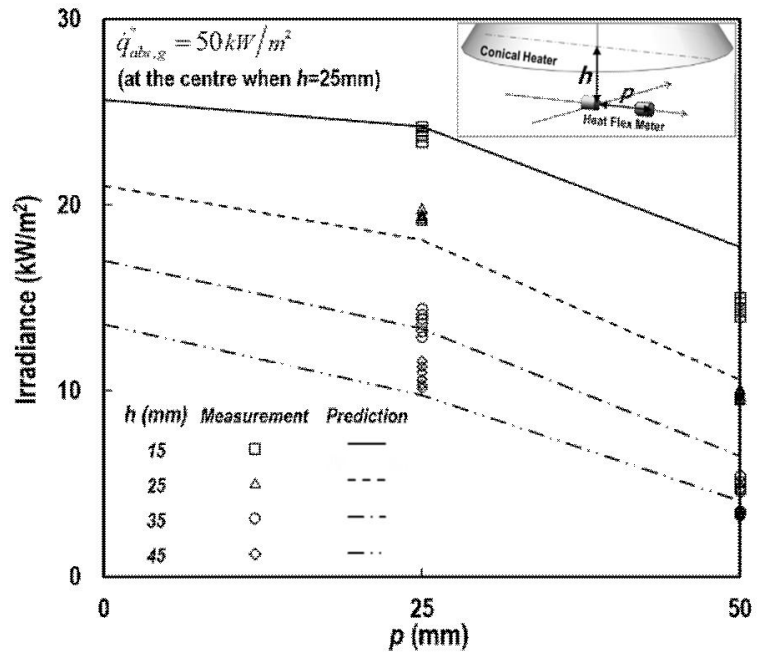

(b) outward-facing surface

Fig. 8. Comparisons between irradiance data sets theoretically predicted and physically measured. 


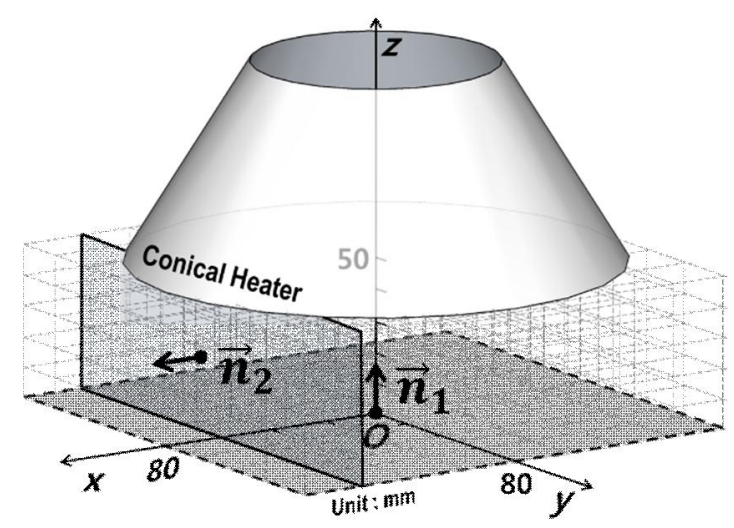

Fig. 9. A schematic of the defined local coordinate system underneath the conical heater.

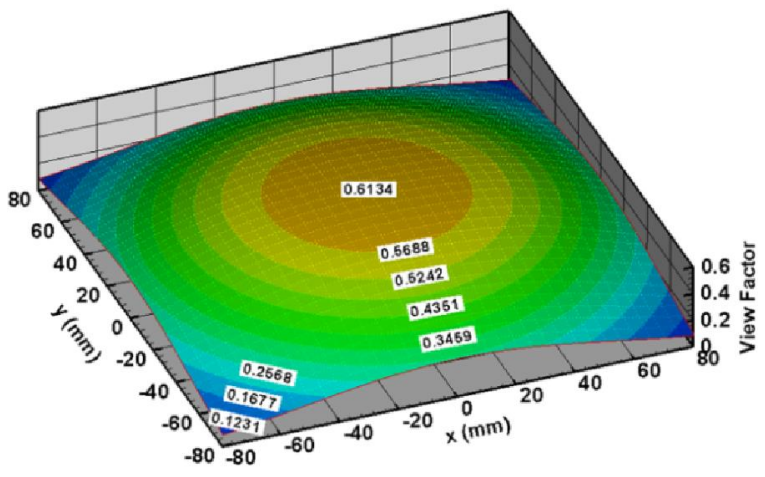

(a) $z=0 \mathrm{~mm}$

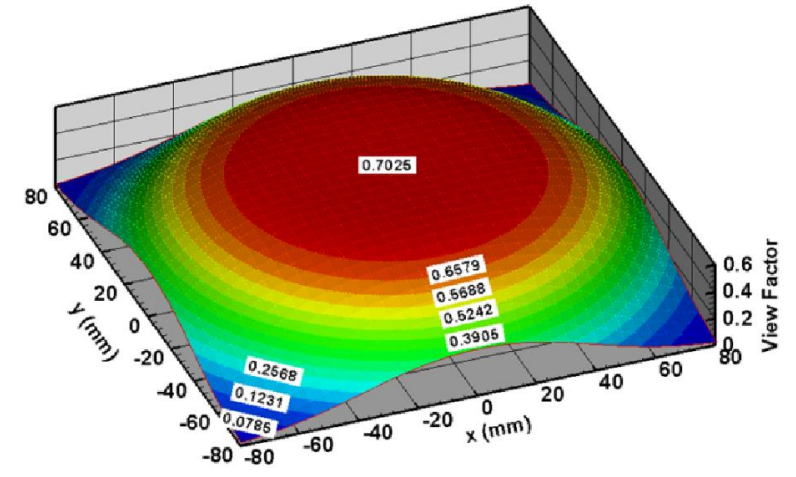

(b) $z=25 \mathrm{~mm}$

Fig. 10. Contour maps of $F_{d A 1-A 3}$. 

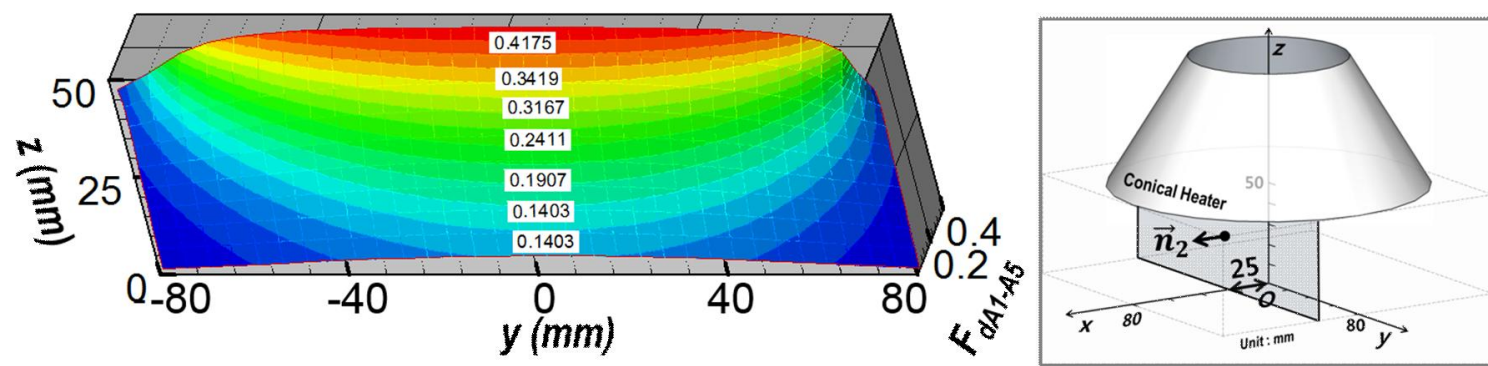

(a) $x=25 \mathrm{~mm}$
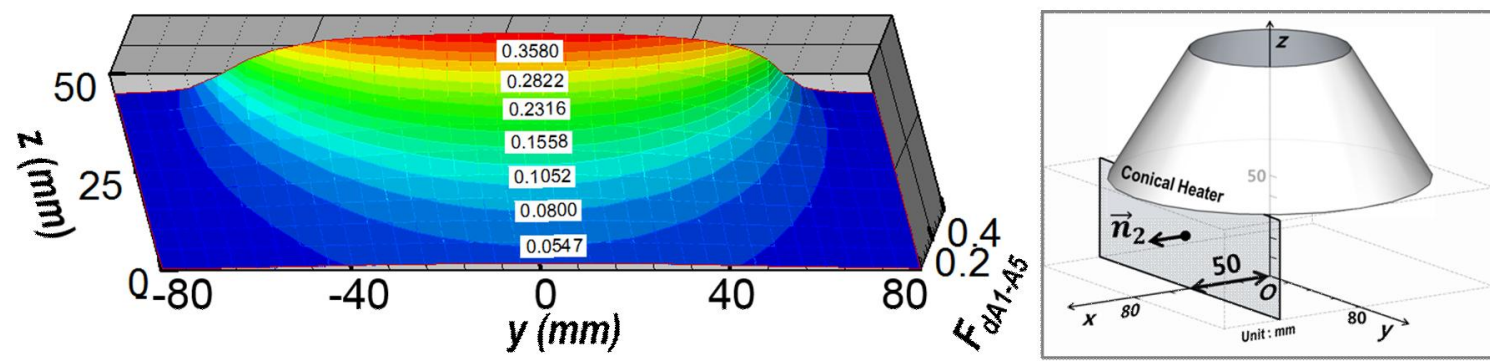

(b) $x=50 \mathrm{~mm}$

Fig. 11. Contour maps of $F_{d A 1-A 5}$.

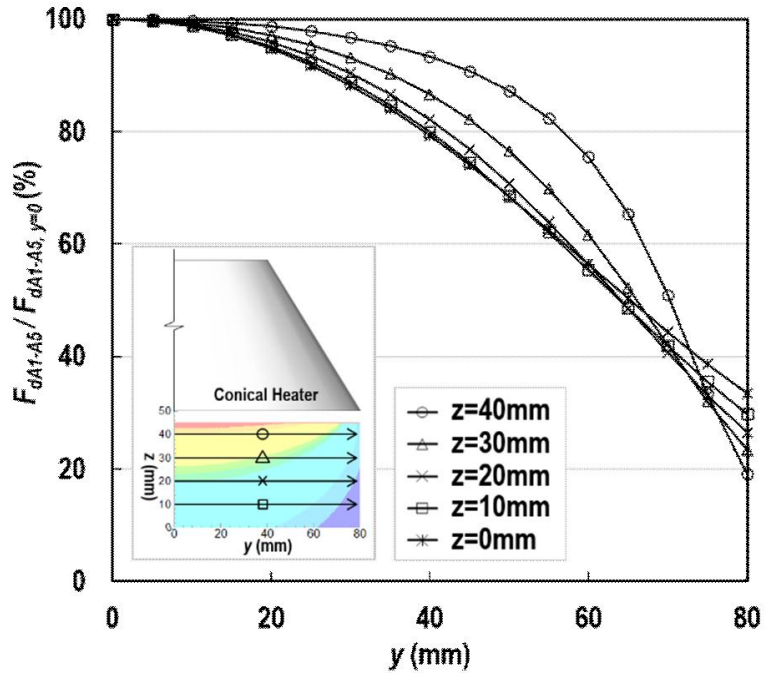

(a) $x=25 \mathrm{~mm}$

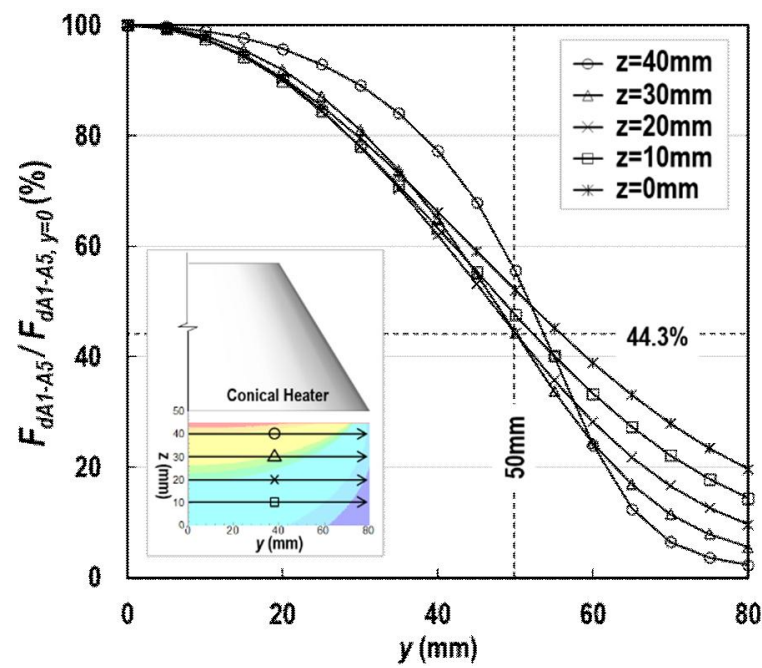

(b) $x=50 \mathrm{~mm}$

Fig. 12. View factor variations in the $y$-direction. 


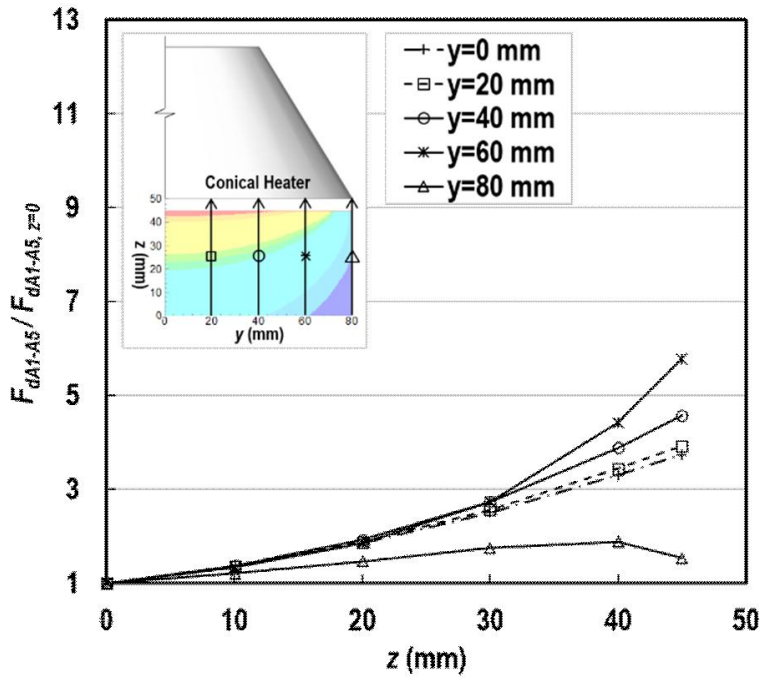

(a) $x=25 \mathrm{~mm}$

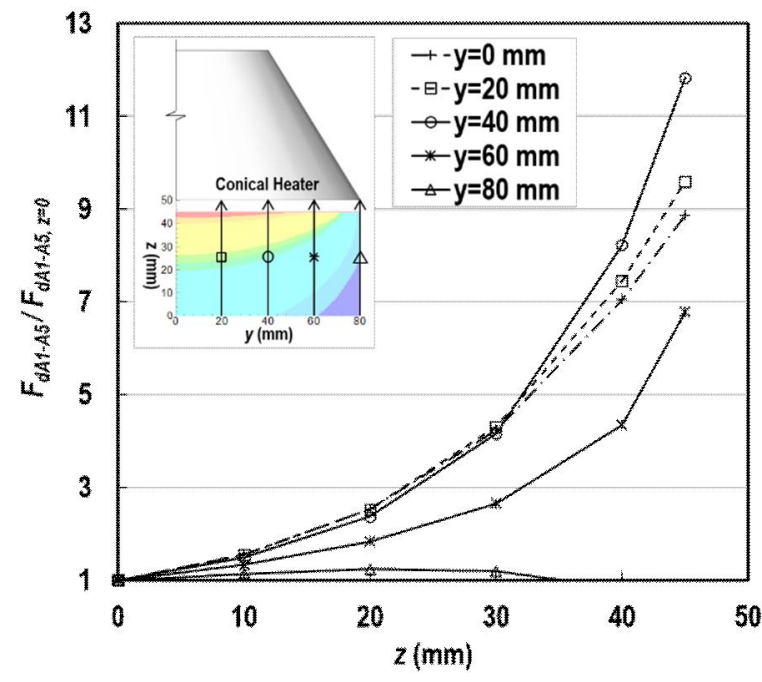

(b) $x=50 \mathrm{~mm}$

Fig. 13. View factor variations in the $z$-coordinate.

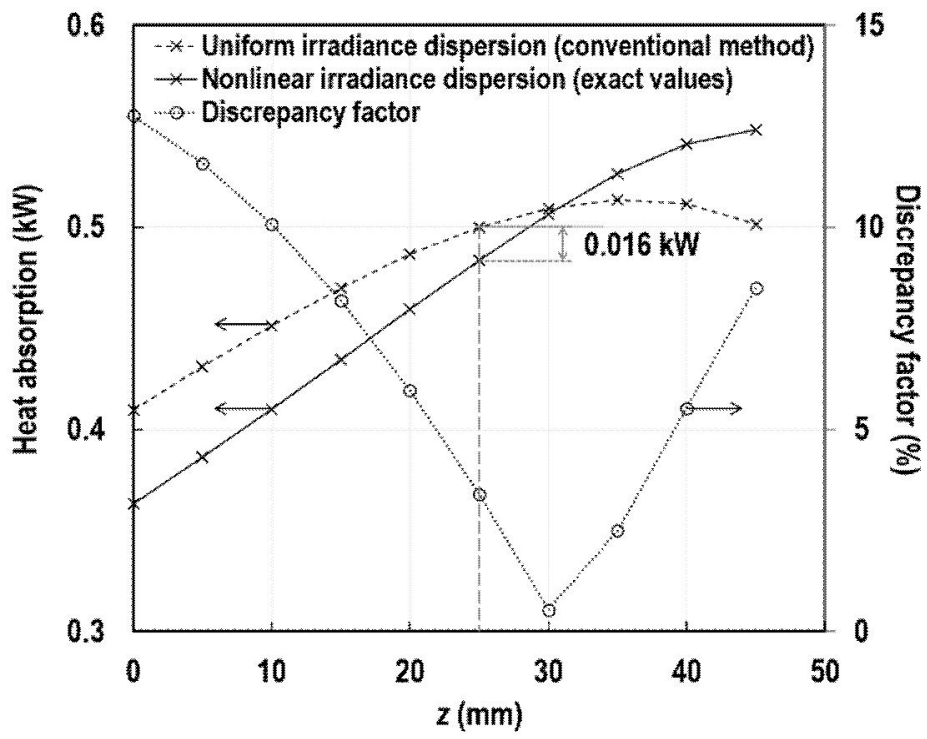

Fig. 14. Comparisons between the quantities of heat absorbed by specimens' top surfaces, and discrepancy factor. 


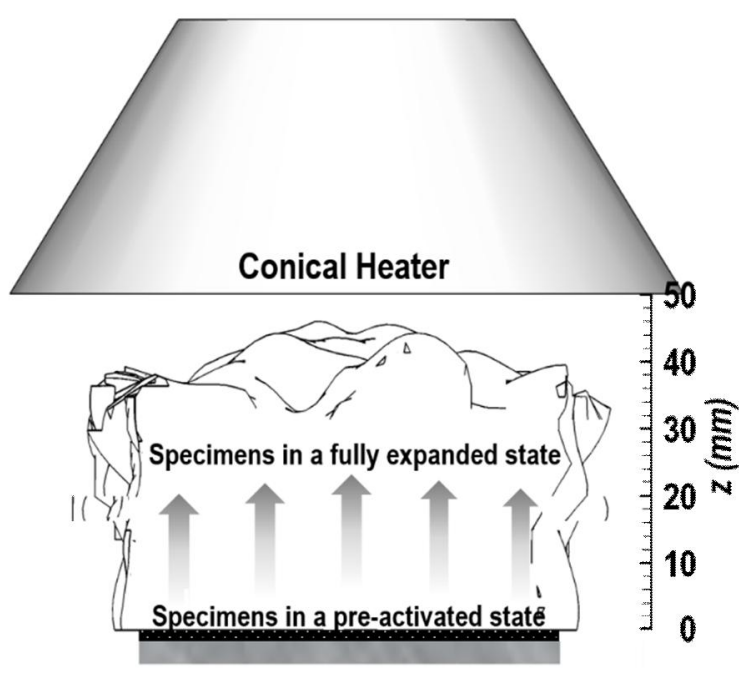

(a) Intumescence

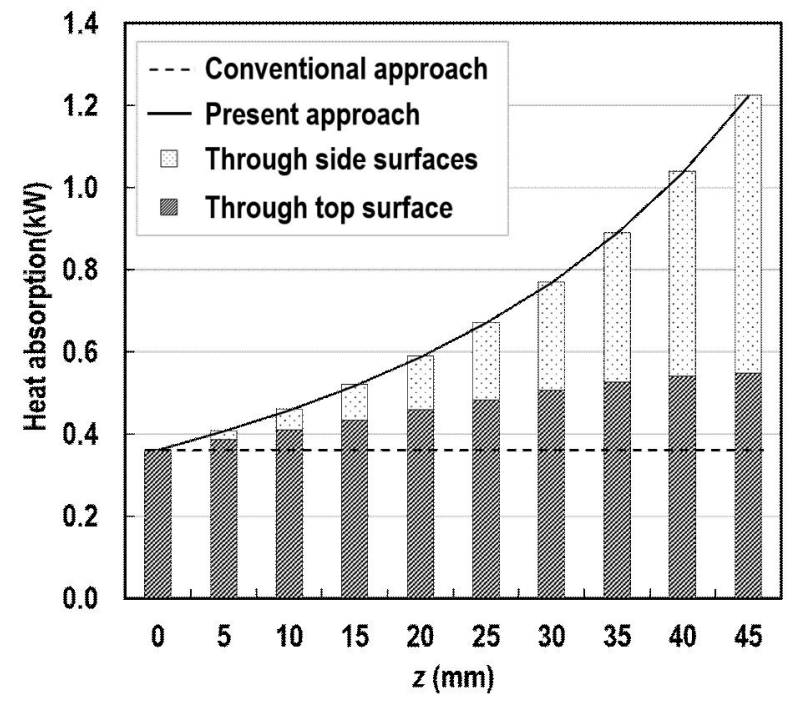

(b) Heat absorption

Fig. 15. Variations in the radiant heat absorbed by a typical intumescent-type sample, developed as it undergoes volumetric progressions in cone calorimeter tests. 